\title{
Vascular Calcification in End-Stage Renal Disease
}

\author{
Kristen L. Jablonski, Ph.D. and Michel Chonchol, M.D. \\ Division of Renal Diseases and Hypertension, University of Colorado Denver Anschutz Medical \\ Center, Aurora, CO 80045
}

\begin{abstract}
Vascular calcification is highly prevalent in end-stage renal disease (ESRD) and independently predictive of future cardiovascular events and mortality. Calcification can occur in both the intimal and medial layers of vasculature, but medial calcification is the major form in ESRD. Medial calcification increases large elastic artery stiffness and pulse-pressure, promotes left ventricular hypertrophy, reduces perfusion of the coronary arteries, and ultimately promotes increased cardiovascular mortality via increased risk of myocardial infarction and heart failure. It results not from a passive deposition of calcium and phosphate due to increased circulating levels, but rather is an active cell-mediated process involving vascular smooth muscle cells (VSMC) apoptosis and vesicle release, a shift in the balance of inhibitors and promoters of vascular calcification, and VSMC differentiation from a contractile to osteochondrogenic phenotype. This phenotypic shift requires phosphate, as well as the uptake of phosphate by the sodium-dependent phosphate cotransporter PiT-1, which is up-regulated by pro-inflammatory cytokines and the uremic milieu. Further research is needed to determine if targeting these processes can ultimately reduce vascular calcification in this high cardiovascular risk population.
\end{abstract}

\section{Keywords}

calcium; end-stage renal disease; phosphate; vascular calcification

\section{Introduction}

Vascular calcification is highly prevalent in end-stage renal disease (ESRD) and independently predictive of future cardiovascular events and mortality. Once thought to simply be a passive process of ectopic calcium deposition resulting from an increased calcium phosphate product $\left(\mathrm{Ca}^{2+} \times \mathrm{P}^{2-}\right)$, calcification is now recognized as a complex active process involving key regulatory proteins typically involved in bone formation and structure that become expressed in arterial tissue and lead to ossification of the vasculature. In this review, we briefly discuss the significance of and mechanisms mediating vascular calcification in ESRD.

\section{Discussion}

\section{Vascular Calcification and Cardiovascular Disease in ESRD}

Cardiovascular mortality risk is elevated 5-10 fold in ESRD compared to the general population (1). An important risk factor for cardiovascular mortality in ESRD patients is

Correspondence to:, Kristen Jablonski, Ph.D., Division of Renal Diseases and Hypertension, 12700 East $19^{\text {th }}$ Avenue C281, Aurora, CO 80045, Phone: (303) 724-4842, Fax: (303 724-4868, Kristen.Nowak@ucdenver.edu.

Disclosures

None. 
vascular calcification, which is an abnormal deposition of calcium salts in vascular tissue, including valves, blood vessels and the heart $(2,3)$. Vascular calcification is highly prevalent in ESRD patients, occurs decades earlier than in the general population (4), and its progression accelerates dramatically once a patient initiates chronic dialysis (5). This is of great clinical significance, as the presence and degree of calcification independently predicts future cardiovascular events, as well as mortality $(6,7)$.

Calcification can occur in both the intimal and medial layers of vasculature, but medial calcification is considered the more common and major form of calcification in ESRD (2). Intimal calcification is focal, associated with inflammation, and is reflective of the degree of atherosclerosis in the aorta, coronary arteries and large vessels $(8,9)$. Medial calcification, also known as Monckeberg's sclerosis, occurs with aging, diabetes and chronic kidney disease (2), is characterized by diffuse mineral deposition throughout the vascular tree and is common in the muscular conduit arteries such as the femoral artery (10). It can occur independent of both atherosclerosis and an increased calcium and phosphorus serum concentration, and results from vascular smooth muscle cell (VSMC) phenotypic changes promoting a upregulation of osteogenic programs (11).

Medial calcification is associated with increased large-elastic artery stiffness, as reflected by increased aortic pulse-wave velocity (Figure 1) (2). This reduction in arterial distensiblity increases systolic blood pressure (SBP) and reduces (DBP), increases cardiac afterload, and compromises perfusion of the coronary arteries during diastole. These consequences are augmented further if coronary plaque is also present (12). Subsequent left ventricular remodeling and hypertrophy increases risk of myocardial infarction and heart failure, and ultimately favors increased cardiovascular mortality (13).

Medial calcification occurs in young to middle-aged patients on chronic dialysis even in absence of traditional cardiovascular risk factors (14). In fact, the extent of coronary artery calcification in dialysis patients is $2-5$ fold greater in age-matched patients who have angiographically proven coronary artery disease (15). Thus, non-traditional risk factors unique to the ESRD patients on chronic dialysis may predispose this population to accelerated vascular calcification (16). However, it is worth noting that although extremely common, not all patients develop vascular calcification despite similar exposure to an uremic milieu, which suggests that genetics and/or protective mechanisms may be important factors (10).

\section{Mechanisms Mediating Vascular Calcification in ESRD}

Normal vessels and valve do not calcify, despite a serum concentration of calcium and phosphorus at or above the solubility product (2), and calcification can still occur even with tight phosphate control (10). Thus, although vascular calcification was once considered to be a passive process of mineral deposition, high serum calcium and phosphorus concentration alone will not result in vascular calcification (17). Instead, calcification is an active cellmediated process involving key regulators that are usually only involved in bone formation becoming expressed in the vasculature and promoting ossification (18). While an increased calcium and phosphate are not sufficient to promote vascular calcification, they are key to both the initiation and progression of calcification (15).

Although calcium and phosphate work synergistically to promote vascular calcification, it is thought that calcium acts primarily by inducing apoptosis of VSMCs (Figure 2). This process promotes release of membrane-bound matrix vesicles that act with the apoptotic VSMCS as nidus for further calcium and phosphate (hydoxyapatite deposition) (17) and promote further apoptosis (19). Vessels from dialysis patients contain almost twice the calcium load of non-dialysis dependent chronic kidney disease patients, and are 
characterized by apoptotic VSMC damage and vesicle release (17). Increased VSMC apoptosis is observed even in pediatric dialysis patients, who are free from traditional cardiovascular risk factors (20). Concurrent with VSMC apoptosis, phenotypic changes also occur in VSMCs.

VSMCs are mesenchymal in origin and thus can differentiate into other mesenchymalderived cells under conditions of stress (17). Differentiation of contractile VSMCs into a chondroycte or osteoblast-like cells is key to vascular calcification and is promoted by active inducers. Ordinarily, key inhibitors counter this process, but this balance is shifted in ESRD (Figure 2). Active inducers that are likely up-regulated include bone morphogenic protein 2 (BMP-2), a key inducer of the differentiation of VSMCs to osetoblast-like cells, receptor activator of nuclear factor kappa B ligand (RANKL), a trans-membrane protein that promotes osteoclast differentiation and activation, and core binding factor alpha 1, runtrelated transcription factor 2 (CFBA1/RUNX2), an essential transcription factor for osteoblast differentiation and osteogenesis (16). Expression of osteochondrogenic genes is also increased even in arteries from pediatric dialysis patients (20).

These active inducers are ordinarily countered by active inhibitors of vascular calcification, which are down-regulated in ESRD. These include matrix gla protein, a transcription factors that inhibits VSMC differentiation to an osteoblast-like phenotype as well as calcium crystal binding in the vascular matrix, osteopontin, a promoter of osteoclast function and inhibitor of hydroxyapatite crystal growth, fetuin-A, one of the most potent inhibitors of ectopic calcification and VSMC apoptosis, osteoprotegerin, an inhibitor of osteoclast differentiation, and BMP-7, a promoter of the VSMC phenotype (16). Fetuin-A levels are low in patients on chronic dialysis and associate with increased coronary artery calcification and cardiovascular mortality $(15,21)$.

Rodent models of renal insufficiency have demonstrated that the phenotypic change in VSMCs from a contractile to an osteochondrogenic phenotype, resulting from this shift in balance of active inhibitors and active promoters, requires high phosphate feeding (22). Phosphate transport into cells is primarily mediated by sodium-dependent phosphate cotransporters, with PiT-1 being most important in VSMCs (2). PiT-1 is required in vitro for differentiation to an osteochondrogenic phenotype and calcification to occur in human aortic smooth muscle cells $(2,23)$. In ESRD, pro-inflammatory cytokines and the uremic milieu can increase PiT-1 expression in VSMCs, leading to increased uptake of phosphorus even if levels are still normal, and further promoting vascular calcification (2).

\section{Summary}

In summary, vascular calcification is highly prevalent in the dialysis population and a major contributor to the increased risk of cardiovascular mortality. It results not simply from a passive deposition of calcium and phosphate, but rather from active processes involving VSMC apoptosis, a shift in the balance of inhibitors and promoters, and VSMC differentiation from a contractile to osteochondrogenic phenotype. Future research is required to determine if targeting these processes can ultimately reduce vascular calcification in this high cardiovascular risk population.

\section{Acknowledgments}

This work is supported by National Institute of Diabetes and Digestive and Kidney Disease award R01DK094796-01 and the American Heart Association award 12POST11920023. 


\section{References}

1. Foley RN, Parfrey PS. Cardiovascular disease and mortality in ESRD. Journal of nephrology. 1998; 11:239-245. [PubMed: 9831236]

2. Giachelli CM. The emerging role of phosphate in vascular calcification. Kidney international. 2009; 75:890-897. [PubMed: 19145240]

3. Verberckmoes SC, Persy V, Behets GJ, et al. Uremia-related vascular calcification: more than apatite deposition. Kidney international. 2007; 71:298-303. [PubMed: 17149373]

4. Goodman WG, Goldin J, Kuizon BD, et al. Coronary-artery calcification in young adults with endstage renal disease who are undergoing dialysis. The New England journal of medicine. 2000; 342:1478-1483. [PubMed: 10816185]

5. Foley RN, Parfrey PS, Sarnak MJ. Clinical epidemiology of cardiovascular disease in chronic renal disease. American journal of kidney diseases: the official journal of the National Kidney Foundation. 1998; 32:S112-119. [PubMed: 9820470]

6. Blacher J, Guerin AP, Pannier B, et al. Arterial calcifications, arterial stiffness, and cardiovascular risk in end-stage renal disease. Hypertension. 2001; 38:938-942. [PubMed: 11641313]

7. London GM, Guerin AP, Marchais SJ, et al. Arterial media calcification in end-stage renal disease: impact on all-cause and cardiovascular mortality. Nephrology, dialysis, transplantation: official publication of the European Dialysis and Transplant Association - European Renal Association. 2003; 18:1731-1740.

8. Guerin AP, London GM, Marchais SJ, et al. Arterial stiffening and vascular calcifications in endstage renal disease. Nephrology, dialysis, transplantation: official publication of the European Dialysis and Transplant Association - European Renal Association. 2000; 15:1014-1021.

9. Abedin M, Tintut Y, Demer LL. Vascular calcification: mechanisms and clinical ramifications. Arteriosclerosis, thrombosis, and vascular biology. 2004; 24:1161-1170.

10. Covic A, Kanbay M, Voroneanu L, et al. Vascular calcification in chronic kidney disease. Clinical science. 2010; 119:111-121. [PubMed: 20443781]

11. Hruska KA, Saab G, Mathew S, et al. Renal osteodystrophy, phosphate homeostasis, and vascular calcification. Seminars in dialysis. 2007; 20:309-315. [PubMed: 17635820]

12. Guerin AP, Blacher J, Pannier B, et al. Impact of aortic stiffness attenuation on survival of patients in end-stage renal failure. Circulation. 2001; 103:987-992. [PubMed: 11181474]

13. London GM. Alterations of arterial function in end-stage renal disease. Nephron. 2000; 84:111118. [PubMed: 10657710]

14. Toussaint ND, Kerr PG. Vascular calcification and arterial stiffness in chronic kidney disease: implications and management. Nephrology. 2007; 12:500-509. [PubMed: 17803475]

15. Moe SM, Chen NX. Pathophysiology of vascular calcification in chronic kidney disease. Circulation research. 2004; 95:560-567. [PubMed: 15375022]

16. Kendrick J, Chonchol M. The role of phosphorus in the development and progression of vascular calcification. American journal of kidney diseases: the official journal of the National Kidney Foundation. 2011; 58:826-834. [PubMed: 21956015]

17. Shroff R, Long DA, Shanahan C. Mechanistic insights into vascular calcification in CKD. Journal of the American Society of Nephrology: JASN. 2013; 24:179-189. [PubMed: 23138485]

18. Mizobuchi M, Towler D, Slatopolsky E. Vascular calcification: the killer of patients with chronic kidney disease. Journal of the American Society of Nephrology: JASN. 2009; 20:1453-1464. [PubMed: 19478096]

19. Olszak IT, Poznansky MC, Evans RH, et al. Extracellular calcium elicits a chemokinetic response from monocytes in vitro and in vivo. The Journal of clinical investigation. 2000; 105:1299-1305. [PubMed: 10792005]

20. Shroff RC, McNair R, Figg N, et al. Dialysis accelerates medial vascular calcification in part by triggering smooth muscle cell apoptosis. Circulation. 2008; 118:1748-1757. [PubMed: 18838561]

21. Ketteler M, Bongartz P, Westenfeld R, et al. Association of low fetuin-A (AHSG) concentrations in serum with cardiovascular mortality in patients on dialysis: a cross-sectional study. Lancet. 2003; 361:827-833. [PubMed: 12642050] 
22. El-Abbadi MM, Pai AS, Leaf EM, et al. Phosphate feeding induces arterial medial calcification in uremic mice: role of serum phosphorus, fibroblast growth factor-23, and osteopontin. Kidney international. 2009; 75:1297-1307. [PubMed: 19322138]

23. Shanahan CM, Crouthamel MH, Kapustin A, et al. Arterial calcification in chronic kidney disease: key roles for calcium and phosphate. Circulation research. 2011; 109:697-711. [PubMed: 21885837] 


\section{Vascular Calcification}

Arterial Stiffness

$\downarrow$

Increased SBP

Decreased DBP

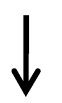

Increased Afterload

$\downarrow$

Reduced Coronary

Perfusion

$\downarrow$

Left Ventricular Hypertrophy

$\downarrow$

Increased MI Risk

Heart Failure

$\downarrow$

\section{Increased CV Mortality}

Figure 1.

Cardiovascular implications of vascular calcification.

Medial vascular calcification increased aortic stiffness (decreased arterial compliance), as reflected by increased aortic pulse-wave velocity. This reduction in arterial distensiblity increases systolic blood pressure (SBP) and also reduces (DBP). Such hemodynamic alterations increases cardiac afterload, while also compromising perfusion of the coronary arteries. Subsequent left ventricular remodeling and hypertrophy increases risk of myocardial infarction (MI) and heart failure, and ultimately favors increased cardiovascular mortality. 


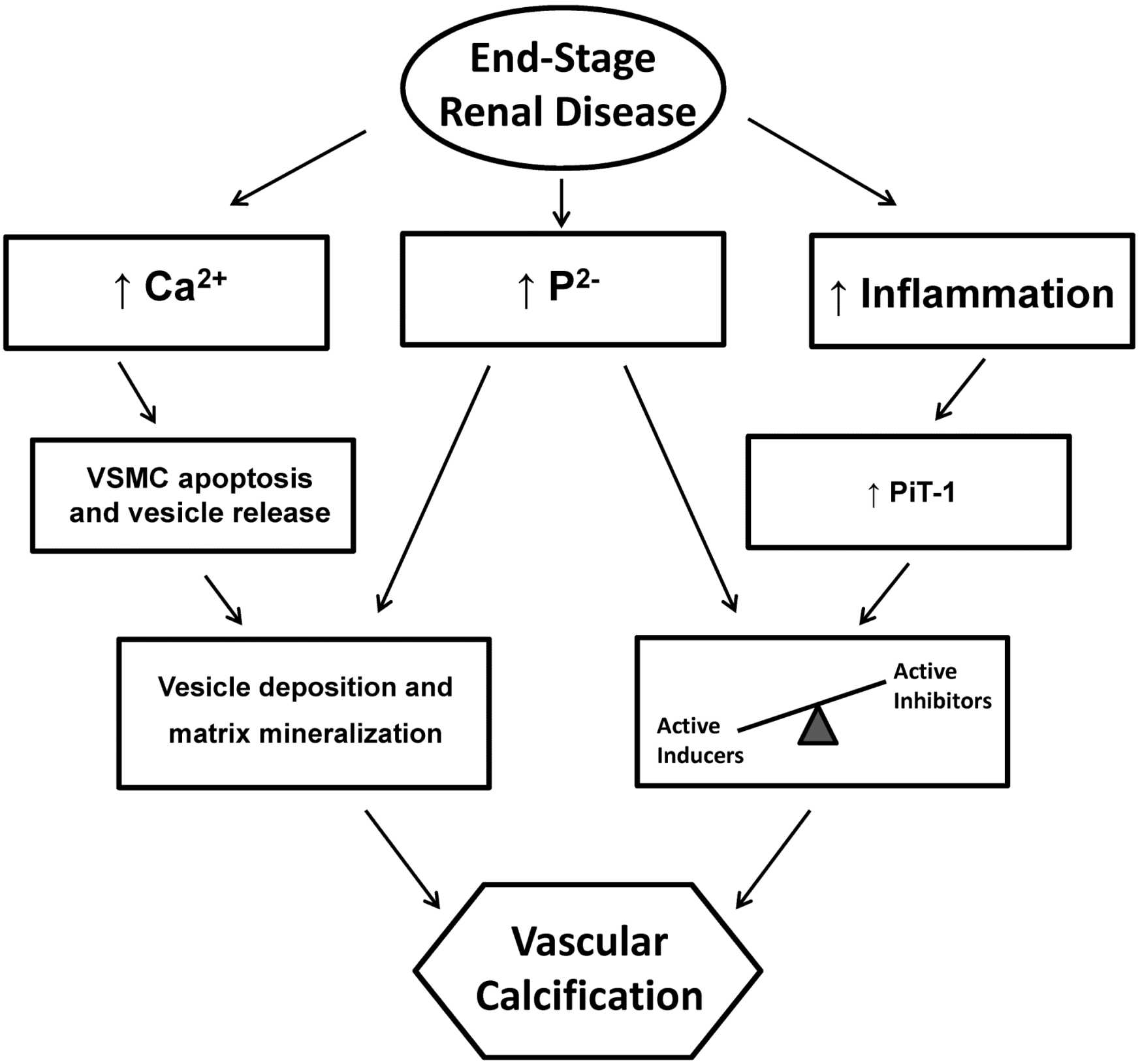

Figure 2.

Simplified diagram of the passive and active processes involved in vascular calcification in end-stage renal disease.

Vascular calcification is not just a passive process resulting from an increased in serum calcium and phosphorus concentration but active process involving key regulatory proteins typically involved in bone formation and structure that become expressed in arterial tissue and lead to ossification of the vasculature. In end-stage renal disease (ESRD), both serum calcium and phosphorous are elevated, and although they work synergistically to promote vascular calcification, it is thought that calcium primarily induces apoptosis, leading to the formation of a mineral nucleation nidus, and phosphorous promotes active osteochondrosis. Calcium promotes vascular smooth muscle cell (VSMC) apoptosis and release of membrane-bound matrix vesicles, leading to vesicle deposition and extracellular matrix mineralization. Subsequently, phenotypic changes in VSMCs occur, a process requiring a 
shift in the balance of cell-mediated processes regulated by active inhibitors and active inducers. Pro-inflammatory cytokines (increased inflammation) and the uremic milieu can increase expression of sodium-dependent phosphate cotransporters (in particular PiT-1) in VSMCs, leading to increased uptake of phosphorus even if levels are still normal. PiT-1 is required for the osteochondrogenic phenotypic changes that occur in VSMC as a result of active processes. 